\title{
DEGREE OF THE CANONICAL MAP OF A GORENSTEIN 3-FOLD OF GENERAL TYPE
}

\author{
JIN-XING CAI \\ (Communicated by Ted Chinburg)
}

\begin{abstract}
We prove that, for a complex projective Gorenstein 3-fold $X$ of general type with locally factorial terminal singularities, if $p_{g}(X)>105411$ and the canonical map $\phi_{X}$ of $X$ is generically finite, then $\operatorname{deg} \phi_{X} \leq 72$.
\end{abstract}

\section{INTRODUCTION}

Let $X$ be a complex projective Gorenstein 3-fold of general type with locally factorial terminal singularities. In [Ha], C. Hacon proved that, if the canonical map $\phi_{X}$ of $X$ is generically finite, then $\operatorname{deg} \phi_{X} \leq 576$. Recall that for a smooth surface $S$ of general type, if the canonical map $\phi_{S}$ of $S$ is generically finite, then $\operatorname{deg} \phi_{S} \leq 36$, and $\operatorname{deg} \phi_{S} \leq 9$ provided $p_{g}(S) \geq 30$ [Be]. By analogy with the case of surfaces, an interesting problem is to give the best bound for the degree of the canonical map of $X$ when $p_{g}(X)$ is sufficiently large. The aim of this note is to prove the following.

Theorem 1.1. Let $X$ be a complex projective Gorenstein 3-fold of general type with locally factorial terminal singularities. Assume that $p_{g}(X)>105411$, and that the canonical map $\phi_{X}$ of $X$ is generically finite. Then $\operatorname{deg} \phi_{X} \leq 72$.

Remark 1.2. (i) The assumption that $X$ is Gorenstein is indispensable for Theorem 1.1 by Hacon's example [Ha, Example 2.1], and also that $p_{g}(X)>105411$ is technical.

(ii) The bound in Theorem 1.1 seems to be the best possible. See Remark 3.5 below for a discussion.

Theorem 1.1 is proved in Section 3. Thanks to Hacon's argument in [Ha], we reduce the problem to the case when the Albanese map of $X$ is a fibration $f: X \rightarrow C$ over a curve $C$, with $g(C)$ being sufficiently large and $p_{g}(F)$ being small, where $F$ is a general fiber of $f$. In this case, we have $\operatorname{deg} \phi_{X} \leq \operatorname{deg} \phi_{X \mid F} \cdot \operatorname{deg} \phi_{\mathcal{L}}$ for any invertible subsheaf $\mathcal{L} \subset f_{*} \omega_{X}$ with $h^{0}(\mathcal{L}) \geq 2$, where $\omega_{X}$ is the dualizing sheaf of $X$ and $\phi_{\mathcal{L}}$ is the rational map associated to $\mathcal{L}$. So the main point is to show that there exists an invertible subsheaf $\mathcal{L} \subset f_{*} \omega_{X}$ with higher degree compared with $g(C)$, which is done in Section 2.

Received by the editors September 13, 2006 and, in revised form, February 21, 2007. 2000 Mathematics Subject Classification. Primary 14J30, 14E35. 


\section{TEChnical preparations}

2.1. Let $X$ be a smooth complex projective variety of general type, $f: X \rightarrow C$ a fiber space over a smooth projective curve $C$, and $F$ a general fiber of $f$. Assume that the canonical map $\phi_{X}$ of $X$ is generically finite. Then we have that $p_{g}(F) \geq$ $\operatorname{dim} X$. (If $p_{g}(F)<\operatorname{dim} X$, then the dimension of the image of the restriction of $\phi_{X}$ to $F$ is at most $\operatorname{dim} X-2$, and hence $\operatorname{dim} \operatorname{Im} \phi_{X} \leq \operatorname{dim} X-1$, which contradicts the assumption that $\phi_{X}$ is generically finite.)

Let $\Sigma \subset \mathbb{P}^{p_{g}(X)-1}$ be the image of $\phi_{X}$. Desingularizing $\Sigma$ and blowing-up $X$ if necessary, we can assume that $\Sigma$ is smooth and $\phi_{X}$ is a morphism. Let $H$ be a very ample divisor on $C$, and $H_{i} \in|H|(i=1,2)$ such that $\operatorname{supp} H_{1} \cap \operatorname{supp} H_{2}=\emptyset$. Let $D_{i}=\phi_{X *}\left(f^{*} H_{i}\right)$. We have that $D_{1}$ is linearly equivalent to $D_{2}$. Let $\alpha^{\prime}: \Sigma \rightarrow \mathbb{P}^{1}$ be the rational map associated to the pencil generated by $D_{1}$ and $D_{2}$. Blowing-up $\Sigma$ and $X$ if necessary, we can assume that $\alpha^{\prime}$ and $\phi_{X}$ are morphisms. Let $\alpha: \Sigma \rightarrow B$ be the Stein factorization of $\alpha^{\prime}$. By construction, we have that for each point $p \in B,\left(\alpha \circ \phi_{X}\right)^{*} p$ is contained in some union of fibers of $f$. So there is a morphism $\pi: C \rightarrow B$ such that the diagram

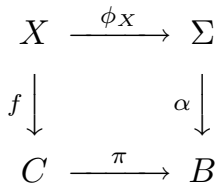

is commutative. We have

$$
\operatorname{deg} \phi_{X}=\operatorname{deg} \phi_{X \mid F} \operatorname{deg} \pi .
$$

Lemma 2.2. Let $n \geq 2$ be an integer, and let $p_{1}, p_{2}, \cdots, p_{n}$ be different points of $C$ with $f^{*} p_{i}$ smooth for all $i$. Assume that $h^{1}\left(f_{*} \omega_{X}\right)=0$ and

$$
h^{1}\left(f_{*} \omega_{X} \otimes \mathcal{O}_{C}\left(-\sum_{i=1}^{n} p_{i}\right)\right) \leq(n-1) p_{g}(F)-1 .
$$

Then $\pi\left(p_{i}\right) \neq \pi\left(p_{j}\right)$ for some $1 \leq i, j \leq n$ (in other words, $\phi_{X}$ separates $f^{*} p_{i}$ and $\left.f^{*} p_{j}\right)$.

Proof. For any subset $I \subset N:=\{1,2, \cdots, n\}$, consider the exact sequence of sheaves

$$
0 \rightarrow f_{*} \omega_{X} \otimes \mathcal{O}_{C}\left(-\sum_{i \in I} p_{i}\right) \rightarrow f_{*} \omega_{X} \rightarrow \bigoplus_{i \in I}\left(f_{*} \omega_{X} \otimes \mathcal{O}_{p_{i}}\right) \rightarrow 0 .
$$

Taking cohomology, we get the long exact sequence

$$
\begin{aligned}
& 0 \rightarrow H^{0}\left(f_{*} \omega_{X} \otimes \mathcal{O}_{C}\left(-\sum_{i \in I} p_{i}\right)\right) \rightarrow H^{0}\left(f_{*} \omega_{X}\right) \stackrel{r_{I}}{\longrightarrow} \bigoplus_{i \in I} H^{0}\left(f_{*} \omega_{X} \otimes \mathcal{O}_{p_{i}}\right) \\
& \rightarrow H^{1}\left(f_{*} \omega_{X} \otimes \mathcal{O}_{C}\left(-\sum_{i \in I} p_{i}\right)\right) \rightarrow H^{1}\left(f_{*} \omega_{X}\right) \rightarrow \cdots .
\end{aligned}
$$

The assumptions imply that

$$
\operatorname{dim} \operatorname{Im} r_{N} \geq p_{g}(F)+1 .
$$

For any subsets $I, J \subset N$, if $I \subset J$, we have $q_{J, I} \circ r_{J}=r_{I}$, where

$$
q_{J, I}: \bigoplus_{j \in J} H^{0}\left(f_{*} \omega_{X} \otimes \mathcal{O}_{p_{j}}\right) \rightarrow \bigoplus_{i \in I} H^{0}\left(f_{*} \omega_{X} \otimes \mathcal{O}_{p_{i}}\right)
$$

is the projection. For convenience, if $I=\{i\}$, we set $r_{i}=r_{I}$. 
Note that for any non-zero section $s \in H^{0}\left(f_{*} \omega_{X}\right)$, let $D \in\left|K_{X}\right|$ be the divisor corresponding to $s$; we have $r_{I}(s)=0$ if and only if $\sum_{i \in I} f^{*} p_{i}<D$.

We proceed by induction on $n$. If $n=2$, by (2.2.1), we have that for $i=1$ and 2 , there exists a non-zero section $s_{i}$ with $r_{\{1,2\}}\left(s_{i}\right) \neq 0$ and $r_{i}\left(s_{i}\right)=0$. Let $D_{i} \in\left|K_{X}\right|$ be the divisor corresponding to $s_{i}$. Then we have

$$
f^{*} p_{1}<D_{1}, f^{*} p_{1} \nless D_{2}, f^{*} p_{2} \nless D_{1}, f^{*} p_{2}<D_{2} .
$$

Let $h$ be a rational function on $X$ such that $\operatorname{div}(h)=D_{1}-D_{2}$, and let $\phi_{h}: X \rightarrow \mathbb{P}^{1}$ be the rational map associated to $h$. Then for a general point $x_{i} \in f^{*} p_{i}(i=1,2)$, we have $h\left(x_{1}\right)=0$ and $h\left(x_{2}\right)=\infty$. This implies $\pi\left(p_{1}\right) \neq \pi\left(p_{2}\right)$. Indeed, if $\pi\left(p_{1}\right)=\pi\left(p_{2}\right)$, then $\phi_{X}$ maps both $f^{*} p_{1}$ and $f^{*} p_{2}$ onto $\alpha^{*} b$, where $b=\pi\left(p_{1}\right)$ (and $\alpha$ is as in subsection 2.1). For any point $x_{i}^{\prime} \in f^{*} p_{i} \cap \phi_{X}^{-1}(z)(i=1,2)$, where $z \in \alpha^{*} b$ is a given general point, we have $\phi_{X}\left(x_{1}^{\prime}\right)=\phi_{X}\left(x_{2}^{\prime}\right)$, but $h\left(x_{1}^{\prime}\right)=0$ and $h\left(x_{2}^{\prime}\right)=\infty$. This is a contradiction.

If $n>2$, by induction, we may assume that $\operatorname{dim} \operatorname{Im} r_{N \backslash\{i\}} \leq p_{g}(F)$ for all $i \in N$. By (2.2.1), we have that $\operatorname{Ker} q_{N, N \backslash\{i\}} \cap \operatorname{Im} r_{N} \neq 0$ for all $i \in N$. This implies that for each $i \in N$, there exists a non-zero section $s_{i}$ with $r_{i}\left(s_{i}\right) \neq 0$ and $r_{j}\left(s_{i}\right)=0$ for all $j \neq i$. Now by the same argument as in case $n=2$, we get the result.

2.3. We assume that $h^{1}\left(f_{*} \omega_{X}\right)=0$ and $\operatorname{deg} \pi \geq 2$. Let $2 \leq u \leq \operatorname{deg} \pi$ be an integer. Let $b \in B$ be a general point, and $p_{1}, p_{2}, \cdots, p_{\operatorname{deg} \pi} \in C$ the inverse image of $b$ under $\pi$. For any locally free sheaf $\mathcal{E}$ on $C$, we denote by $\mathcal{E}^{\vee}$ the dual of $\mathcal{E}$. By Lemma 2.2,

$$
h^{0}\left(\left(f_{*} \omega_{X / C}\right)^{\vee} \otimes \mathcal{O}_{C}\left(\sum_{i=1}^{u} p_{i}\right)\right)=h^{1}\left(f_{*} \omega_{X} \otimes \mathcal{O}_{C}\left(-\sum_{i=1}^{u} p_{i}\right)\right) \geq(u-1) p_{g}(F),
$$

where $f_{*} \omega_{X / C}=f_{*} \omega_{X} \otimes \omega_{C}^{-1}$. Let $\mathcal{F}^{\prime}$ be the subsheaf of $\left(f_{*} \omega_{X / C}\right)^{\vee} \otimes \mathcal{O}_{C}\left(\sum_{i=1}^{u} p_{i}\right)$ generated by its global sections, and $\mathcal{F}$ the saturation of $\mathcal{F}^{\prime}$ in $\left(f_{*} \omega_{X / C}\right)^{\vee} \otimes$ $\mathcal{O}_{C}\left(\sum_{i=1}^{u} p_{i}\right)$. We have $h^{0}(\mathcal{F}) \geq(u-1) p_{g}(F)$, and there is an exact sequence of locally free sheaves

$$
0 \longrightarrow \mathcal{F} \otimes \mathcal{O}_{C}\left(-\sum_{i=1}^{u} p_{i}\right) \longrightarrow\left(f_{*} \omega_{X / C}\right)^{\vee} \longrightarrow \mathcal{Q} \longrightarrow 0,
$$

where $\mathcal{Q}$ is the quotient sheaf $\left(f_{*} \omega_{X / C}\right)^{\vee} /\left(\mathcal{F} \otimes \mathcal{O}_{C}\left(-\sum_{i=1}^{u} p_{i}\right)\right)$. Taking dual, we get an exact sequence of locally free sheaves

$$
0 \longrightarrow \mathcal{Q}^{\vee} \longrightarrow f_{*} \omega_{X / C} \longrightarrow \mathcal{F}^{\vee} \otimes \mathcal{O}_{C}\left(\sum_{i=1}^{u} p_{i}\right) \longrightarrow 0 .
$$

Here we insert a lemma that will be used in the sequel.

Lemma 2.4. Let $\mathcal{E}$ be a locally free sheaf of rank $r$ on a projective smooth curve $C$ of positive genus.

(i) If $\mathcal{E}$ is generically generated by global sections, then $\operatorname{deg} \mathcal{E} \geq h^{0}(\mathcal{E})-r$.

(ii) If $\mathcal{E}^{\vee} \otimes \mathcal{H}$ is semipositive, where $\mathcal{H}$ is an invertible sheaf of degree $m \geq 1$, then $h^{0}(\mathcal{E}) \leq m r$.

Proof. (i) The assumption on $\mathcal{E}$ implies $h^{0}(\mathcal{E}) \geq r$, $\operatorname{deg} \mathcal{E} \geq 0$, and $\operatorname{deg} \mathcal{E}=0$ holds only when $\mathcal{E} \simeq \mathcal{O}_{C}^{\oplus r}$. If $r=1$, by the Riemann-Roch theorem, we have $h^{0}(\mathcal{E})-1-\operatorname{deg} \mathcal{E}=h^{0}\left(\mathcal{E}^{\vee} \otimes \omega_{C}\right)-g(C) \leq 0$. Now we assume that $r \geq 2$. A non-zero section $s$ in $H^{0}(\mathcal{E})$ induces an exact sequence of locally free sheaves on $C$

$$
0 \rightarrow \mathcal{L} \rightarrow \mathcal{E} \rightarrow \mathcal{F}:=\mathcal{E} / \mathcal{L} \rightarrow 0
$$


with $h^{0}(\mathcal{L})>0$. Note that $\mathcal{F}$ is also generically generated by global sections. By induction on $\operatorname{rank} \mathcal{E}$, we have $\operatorname{deg} \mathcal{F} \geq h^{0}(\mathcal{F})-(r-1)$. So

$$
\operatorname{deg} \mathcal{E}=\operatorname{deg} \mathcal{F}+\operatorname{deg} \mathcal{L} \geq h^{0}(\mathcal{F})-(r-1)+h^{0}(\mathcal{L})-1 \geq h^{0}(\mathcal{E})-r .
$$

(ii) First we assume that $r=1$. We can assume that $h^{0}(\mathcal{E})>0$. We have $\operatorname{deg} \mathcal{E} \geq 0$, and the equality holds only when $\mathcal{E} \simeq \mathcal{O}_{C}$. If $\operatorname{deg} \mathcal{E}=0$, the lemma is trivially true. have

If $\operatorname{deg} \mathcal{E}>0$, then $h^{0}\left(\mathcal{E}^{\vee} \otimes \omega_{C}\right) \leq g(C)-1$. By the Riemann-Roch theorem, we

$$
h^{0}(\mathcal{E})-\operatorname{deg} \mathcal{E}=h^{0}\left(\mathcal{E}^{\vee} \otimes \omega_{C}\right)+1-g(C) \leq 0 .
$$

Since $\mathcal{E}^{\vee} \otimes \mathcal{H}$ is semipositive, we have $\operatorname{deg} \mathcal{E}^{\vee} \otimes \mathcal{H} \geq 0$. So $\operatorname{deg} \mathcal{E} \leq m$. Adding the above two inequalities we get $h^{0}(\mathcal{E}) \leq m$.

Now we assume that $r>1$. We will show the following

Claim. Set $\mathcal{E}_{0}=\mathcal{E}$. Suppose that $h^{0}\left(\mathcal{E}_{0}\right)>m r$. Then for $j=1, \cdots, r-1$, there exist exact sequences of locally free sheaves on $C$,

$$
0 \rightarrow \mathcal{L}_{j} \rightarrow \mathcal{E}_{j-1} \stackrel{\alpha_{j}}{\longrightarrow} \mathcal{E}_{j} \rightarrow 0,
$$

with the following properties:

(a) $\operatorname{deg} \mathcal{L}_{j}>0$ for all $j$;

(b) $\sum_{i=1}^{j} \operatorname{deg} \mathcal{L}_{i} \leq j m$ for $j=1, \cdots, r-1$;

(c) $h^{0}\left(\mathcal{E}_{j}\right)>m(r-j)$ for $j=1, \cdots, r-1$.

Admitting the Claim for the moment, we have

$$
\begin{gathered}
\operatorname{deg} \mathcal{E}_{i-1}=\operatorname{deg} \mathcal{E}_{i}+\operatorname{deg} \mathcal{L}_{i}, \\
h^{0}\left(\mathcal{E}_{i}\right)+h^{0}\left(\mathcal{L}_{i}\right) \geq h^{0}\left(\mathcal{E}_{i-1}\right)
\end{gathered}
$$

for $i=1, \cdots, r-1$. Adding these equalities and inequalities, we get

$$
\operatorname{deg} \mathcal{E}_{0}+h^{0}\left(\mathcal{E}_{r-1}\right)+\sum_{1=1}^{r-1} h^{0}\left(\mathcal{L}_{i}\right) \geq \operatorname{deg} \mathcal{E}_{r-1}+\sum_{1=1}^{r-1} \operatorname{deg} \mathcal{L}_{i}+h^{0}\left(\mathcal{E}_{0}\right) .
$$

Note that for any invertible sheaf $\mathcal{L}$ of positive degree, we have $h^{0}(\mathcal{L}) \leq \operatorname{deg} \mathcal{L}($ see (2.4.1)). Hence

$$
\operatorname{deg} \mathcal{E}_{0} \geq\left(\operatorname{deg} \mathcal{E}_{r-1}-h^{0}\left(\mathcal{E}_{r-1}\right)\right)+\sum_{1=1}^{r-1}\left(\operatorname{deg} \mathcal{L}_{i}-h^{0}\left(\mathcal{L}_{i}\right)\right)+h^{0}\left(\mathcal{E}_{0}\right) \geq h^{0}\left(\mathcal{E}_{0}\right)>m r,
$$

which contradicts the semi-positive property of $\mathcal{E}_{0}^{\vee} \otimes \mathcal{H}$.

It remains to prove the Claim. We proceed by induction on $j$. Let $p \in C$ be a point. Consider the exact sequence of sheaves

$$
0 \rightarrow \mathcal{E}_{0} \otimes \mathcal{O}_{C}(-p) \rightarrow \mathcal{E}_{0} \rightarrow \mathcal{E}_{0} \otimes \mathcal{O}_{p} \rightarrow 0
$$

Taking cohomology, we get $h^{0}\left(\mathcal{E}_{0} \otimes \mathcal{O}_{C}(-p)\right)>(m-1) r \geq 0$. So there is a non-zero section $s$ in $H^{0}\left(\mathcal{E}_{0} \otimes \mathcal{O}_{C}(-p)\right)$. Then $s$ induces an exact sequence of locally free sheaves on $C$,

$$
0 \rightarrow \mathcal{L}_{1} \rightarrow \mathcal{E}_{0} \stackrel{\alpha_{1}}{\longrightarrow} \mathcal{E}_{1}:=\mathcal{E}_{0} / \mathcal{L}_{1} \rightarrow 0,
$$

with $\operatorname{deg} \mathcal{L}_{1}>0$. Since $\mathcal{E}_{0}^{\vee} \otimes \mathcal{H}$ is semipositive, we have $\operatorname{deg}\left(\mathcal{L}_{1}^{\vee} \otimes \mathcal{H}\right) \geq 0$. So $\operatorname{deg} \mathcal{L}_{1} \leq m$, and hence

$$
h^{0}\left(\mathcal{E}_{1}\right) \geq h^{0}\left(\mathcal{E}_{0}\right)-h^{0}\left(\mathcal{L}_{1}\right)>m r-\operatorname{deg} \mathcal{L}_{1} \geq m(r-1) .
$$


Thus the Claim is true for $j=1$.

Inductively, assume that for $j \leq k$, there exist exact sequences of locally free sheaves on $C$,

$$
0 \rightarrow \mathcal{L}_{j} \rightarrow \mathcal{E}_{j-1} \stackrel{\alpha_{j}}{\longrightarrow} \mathcal{E}_{j} \rightarrow 0
$$

with the above properties (a)-(c). Since $h^{0}\left(\mathcal{E}_{k}\right)>m(r-k)$ (by (c)), by the same argument as above, there exists an exact sequence of locally free sheaves on $C$,

$$
0 \rightarrow \mathcal{L}_{k+1} \rightarrow \mathcal{E}_{k} \stackrel{\alpha_{k}}{\longrightarrow} \mathcal{E}_{k+1}:=\mathcal{E}_{k} / \mathcal{L}_{k+1} \rightarrow 0,
$$

with $\operatorname{deg} \mathcal{L}_{k+1}>0$. Set $\mathcal{F}_{1}=\mathcal{L}_{1}$ and $\mathcal{F}_{i}=\left(\alpha_{i-1} \circ \cdots \circ \alpha_{1}\right)^{-1}\left(\mathcal{L}_{i}\right)$ for $2 \leq i \leq k+1$. Since $\mathcal{L}_{i} \subset \alpha_{i}^{-1}\left(\mathcal{L}_{i+1}\right)$, we have exact sequences of locally free sheaves

$$
0 \rightarrow \mathcal{F}_{i-1} \rightarrow \mathcal{F}_{i} \stackrel{\alpha_{i} \circ \cdots \circ \alpha_{1} \mid \mathcal{F}_{i}}{\longrightarrow} \mathcal{L}_{i} \rightarrow 0
$$

for $i=2, \cdots, k+1$. So

$$
\sum_{i=1}^{k+1} \operatorname{deg} \mathcal{L}_{i}=\sum_{i=2}^{k+1}\left(\operatorname{deg} \mathcal{F}_{i}-\operatorname{deg} \mathcal{F}_{i-1}\right)+\operatorname{deg} \mathcal{L}_{1}=\operatorname{deg} \mathcal{F}_{k+1} \leq(k+1) m
$$

where the last inequality holds since $\mathcal{F}_{k+1}^{\vee} \otimes \mathcal{H}$ is a quotient of $\mathcal{E}^{\vee} \otimes \mathcal{H}$, which is semi-positive. We have $h^{0}\left(\mathcal{E}_{i}\right) \geq h^{0}\left(\mathcal{E}_{i-1}\right)-h^{0}\left(\mathcal{L}_{i}\right) \geq h^{0}\left(\mathcal{E}_{i-1}\right)-\operatorname{deg}\left(\mathcal{L}_{i}\right)$ for $i=1, \cdots, k+1$. Adding these inequalities, we get

$$
h^{0}\left(\mathcal{E}_{k+1}\right) \geq h^{0}\left(\mathcal{E}_{0}\right)-\sum_{i=1}^{k+1} \operatorname{deg} \mathcal{L}_{i}>m r-m(k+1)=m(r-(k+1)) .
$$

So the Claim is true for $j=k+1$. This completes the proof of the Claim.

Lemma 2.5. Notation as in subsection 2.3. We have $\operatorname{rank} \mathcal{Q}^{\vee} \leq \frac{p_{g}(F)}{u}$.

Proof. Set $s=\operatorname{rank} \mathcal{F}$. By Lemma 2.4 (ii), we have

$$
s u \geq h^{0}(\mathcal{F}) \geq(u-1) p_{g}(F) .
$$

So we have $s \geq \frac{(u-1) p_{g}(F)}{u}$, and hence $\operatorname{rank} \mathcal{Q}^{\vee}=p_{g}(F)-s \leq \frac{p_{g}(F)}{u}$.

Lemma 2.6. Let $2 \leq u \leq \operatorname{deg} \pi$ be an integer. There exists an invertible subsheaf $\mathcal{L} \subset f_{*} \omega_{X}$ of degree at least

$$
\min \left\{\left(1+\frac{u}{p_{g}(F)}\right) g(C)+u(u-1)-\frac{\left(p_{g}(F)-2\right)(u+1)}{2}-2,2 g(C)-2-\left(p_{g}(F)-1\right) u\right\} .
$$

Proof. We can assume that $h^{1}\left(f_{*} \omega_{X}\right)=0$; indeed, if $h^{1}\left(f_{*} \omega_{X}\right) \neq 0$, by [Fu, Theorem 3.1], $\mathcal{O}_{C}$ is a direct summand of $f_{*} \omega_{X / C}$. So $\omega_{C} \subset f_{*} \omega_{X}$, and the lemma is trivially true in this case.

Set $t=\operatorname{rank} \mathcal{Q}^{\vee}$. We distinguish two cases according to whether $t \geq 2$ or not. 
Case 1. $t \geq 2$. Let $\mathcal{L}^{\prime} \subset \mathcal{Q}^{\vee}$ be an invertible subsheaf of maximal degree. We have $\operatorname{deg} \mathcal{L}^{\prime} \geq \frac{1}{t} \operatorname{deg} \mathcal{Q}^{\vee}-\frac{t-1}{t} g(C) \quad($ by $[\mathrm{Mu}-\mathrm{Sa}$, Theorem $])$

$$
\begin{aligned}
& \left.=\frac{1}{t}\left(\operatorname{deg} f_{*} \omega_{X / C}-\operatorname{deg} \mathcal{F}^{\vee} \otimes \mathcal{O}_{C}\left(\sum_{i=1}^{u} p_{i}\right)\right)-\frac{t-1}{t} g(C) \quad \text { (by }(2.3 .1)\right) \\
& \geq \frac{1}{t}\left(\operatorname{deg} \mathcal{F}-\left(p_{g}(F)-t\right) u\right)-\frac{t-1}{t} g(C) \quad\left(f_{*} \omega_{X / C} \text { is semipositive [Fu] }\right) \\
& \left.\geq \frac{1}{t}\left((u-1) p_{g}(F)-\left(p_{g}(F)-t\right)(u+1)\right)-\frac{t-1}{t} g(C) \quad \text { (by Lemma 2.4 (i) }\right) \\
& =\frac{1}{t}\left((u-1) p_{g}(F)+g(C)\right)-\frac{\left(p_{g}(F)-t\right)}{t}(u+1)-g(C) \\
& \left.\geq \frac{u}{p_{g}(F)}\left((u-1) p_{g}(F)+g(C)\right)-\frac{\left(p_{g}(F)-2\right)}{2}(u+1)-g(C) \quad \text { by }(2.5)\right) .
\end{aligned}
$$

So $\mathcal{L}:=\mathcal{L}^{\prime} \otimes \omega_{C}$ is an invertible subsheaf of $f_{*} \omega_{X}$ of degree

$$
\operatorname{deg} \mathcal{L} \geq\left(1+\frac{u}{p_{g}(F)}\right) g(C)+u(u-1)-\frac{\left(p_{g}(F)-2\right)(u+1)}{2}-2 .
$$

Case 2. $t \leq 1$. In this case, $\operatorname{rank} \mathcal{F} \geq p_{g}(F)-1$. So we can choose $p_{g}(F)-1$ algebraically independent global sections of $\left(f_{*} \omega_{X / C}\right)^{\vee} \otimes \mathcal{O}_{C}\left(\sum_{i=1}^{u} p_{i}\right)$, and let $\widetilde{\mathcal{F}^{\prime}}$ be the subsheaf of $\left(f_{*} \omega_{X / C}\right)^{\vee} \otimes \mathcal{O}_{C}\left(\sum_{i=1}^{u} p_{i}\right)$ generated by these sections, and $\widetilde{\mathcal{F}}$ the saturation of $\widetilde{\mathcal{F}^{\prime}}$ in $\left(f_{*} \omega_{X / C}\right)^{\vee} \otimes \mathcal{O}_{C}\left(\sum_{i=1}^{u} p_{i}\right)$. Then we have an exact sequence of locally free sheaves

$$
0 \longrightarrow \tilde{\mathcal{L}}^{\prime} \longrightarrow f_{*} \omega_{X / C} \longrightarrow \tilde{\mathcal{F}}^{\vee} \otimes \mathcal{O}_{C}\left(\sum_{i=1}^{u} p_{i}\right) \longrightarrow 0,
$$

where $\widetilde{\mathcal{L}}^{\prime}$ is an invertible sheaf by the choice of $\widetilde{\mathcal{F}}$. We have

$$
\begin{aligned}
\operatorname{deg}{\widetilde{\mathcal{L}^{\prime}}} & =\operatorname{deg} f_{*} \omega_{X / C}-\operatorname{deg} \widetilde{\mathcal{F}}^{\vee} \otimes \mathcal{O}_{C}\left(\sum_{i=1}^{u} p_{i}\right) \\
& =\operatorname{deg} f_{*} \omega_{X / C}+\operatorname{deg} \widetilde{\mathcal{F}}-\left(p_{g}(F)-1\right) u \\
& \geq-\left(p_{g}(F)-1\right) u
\end{aligned}
$$

So $\mathcal{L}:=\widetilde{\mathcal{L}^{\prime}} \otimes \omega_{C}$ is an invertible subsheaf of $f_{*} \omega_{X}$ of degree

$$
\operatorname{deg} \mathcal{L} \geq 2 g(C)-2-\left(p_{g}(F)-1\right) u .
$$

Lemma 2.7. Let $C$ be a smooth projective curve of genus $g \geq 2$, and let $\mathcal{L}$ be an invertible sheaf of positive degree on $C$. If

$$
\operatorname{deg} \mathcal{L}>\left(1+\frac{1}{a}\right)\left(g-h^{1}(\mathcal{L})\right)
$$

for some positive integer $a$, then $\operatorname{deg} \phi_{\mathcal{L}} \leq a$, where $\phi_{\mathcal{L}}$ is the rational map associated to $\mathcal{L}$.

Proof. Since $\operatorname{deg} \mathcal{L}>0$, we have $h^{1}(\mathcal{L})<g$. By the Riemann-Roch theorem and the assumption,

$$
h^{0}(\mathcal{L})-1=\operatorname{deg} \mathcal{L}-\left(g-h^{1}(\mathcal{L})\right)>\frac{1}{a+1} \operatorname{deg} \mathcal{L} .
$$


Hence $h^{0}(\mathcal{L}) \geq 2$. Let $d$ be the degree of the rational map $\phi_{\mathcal{L}}: C \rightarrow \mathbb{P}^{h^{0}(\mathcal{L})-1}$. We have

$$
d\left(h^{0}(\mathcal{L})-1\right)=d\left(\operatorname{codim} \operatorname{Im} \phi_{\mathcal{L}}+1\right) \leq d \operatorname{deg} \operatorname{Im} \phi_{\mathcal{L}} \leq \operatorname{deg} \mathcal{L}
$$

So

$$
d \leq \frac{\operatorname{deg} \mathcal{L}}{h^{0}(\mathcal{L})-1}<a+1
$$

where the last inequality follows by (2.7.1).

Proposition 2.8. Notation as in subsection 2.1. Let $u$, a be integers with $2 \leq$ $u \leq a$ and $u a>p_{g}(F)$. Assume that $g(C)>M\left(u, a, p_{g}(F)\right)$, where we set $M\left(u, a, p_{g}(F)\right)$ to be

$$
\max \left\{\frac{a p_{g}(F)}{u a-p_{g}(F)}\left(2+\frac{\left(p_{g}(F)-2\right)(u+1)}{2}-u(u-1)\right), \quad \frac{a}{a-1}\left(2+\left(p_{g}(F)-1\right) u\right)\right\} .
$$

Then $\operatorname{deg} \pi \leq a$.

Proof. We can assume that $\operatorname{deg} \pi \geq u$; otherwise, the proposition is trivially true. By Lemma 2.6, there exists an invertible subsheaf $\mathcal{L} \subset f_{*} \omega_{X}$ of degree at least

$$
\begin{aligned}
& \min \left\{\left(1+\frac{u}{p_{g}(F)}\right) g(C)+u(u-1)-\frac{\left(p_{g}(F)-2\right)(u+1)}{2}-2,2 g(C)-2-\left(p_{g}(F)-1\right) u\right\} \\
& >\left(1+\frac{1}{a}\right) g(C), \text { provided } g(C)>M\left(u, a, p_{g}(F)\right) .
\end{aligned}
$$

By Lemma 2.7, we get $\operatorname{deg} \phi_{\mathcal{L}} \leq a$.

On the other hand, since $f^{*}|\mathcal{L}|+$ (some effective divisors) is a sublinear system of $\left|K_{X}\right|, \phi_{\mathcal{L}}$ factors through $\pi$, where $\pi$ is as in subsection 2.1. This implies $\operatorname{deg} \pi \leq$ $\operatorname{deg} \phi_{\mathcal{L}}$, and the result follows.

\section{Proof of Theorem 1.1}

3.1. Notation as in Theorem 1.1. Let $d=\operatorname{deg} \phi_{X}$. By the Miyaoka-Yau inequality [M], we have

$$
d\left(p_{g}(X)-3\right) \leq d \operatorname{deg} \operatorname{Im} \phi_{X} \leq K_{X}^{3} \leq 72 \chi\left(\omega_{X}\right),
$$

where the first inequality holds since $\operatorname{Im} \phi_{X}$ is a non-degenerate 3 -fold in $\mathbb{P}^{p_{g}(X)-1}$. So

$$
d \leq 72 \frac{\chi\left(\omega_{X}\right)}{p_{g}(X)-3}
$$

For convenience, we set the right side of (3.1.1) by $d_{X}$.

If $q(X) \leq 1462$, then $\chi\left(\omega_{X}\right) \leq p_{g}(X)+1461$. So $d_{X}<73$ if $p_{g}(X)>105411$ and the result follows in this case.

3.2. Now we can assume hereafter that $q(X) \geq 1463$. Consider the Albanese map $\operatorname{alb}_{X}: X \rightarrow \operatorname{Alb} X$. Let $f: X \rightarrow Y$ be the Stein factorization of alb ${ }_{X}$. By Hacon's argument (see the proof of [Ha, Theorem 1.1]), one has

(3.2.1) $\chi\left(\omega_{X}\right) \leq p_{g}(X)$, if $\operatorname{dim} Y \geq 2$;

(3.2.2) $\chi\left(\omega_{X}\right) \leq p_{g}(X)+\chi\left(\omega_{Y}\right)$ and $\chi\left(\omega_{Y}\right) p_{g}(F) \leq p_{g}(X)$, if $\operatorname{dim} Y=1$.

Hence if $\operatorname{dim} Y \geq 2$, by (3.2.1), $d_{X}<73$ if $p_{g}(X)>219$. 
3.3. Now we assume that $C:=Y$ is a curve (of genus $g(C)=q(X)$ ). Desingularizing $X$ if necessary, we can assume that $X$ is smooth. Let $F$ be a general fiber of $f$. Then $F$ is a surface of general type with $p_{g}(F) \geq 3$ (cf. subsection 2.1). By (3.2.2), we have

$$
d_{X} \leq 72\left(1+\frac{1}{p_{g}(F)}\right) \frac{p_{g}(X)}{p_{g}(X)-3} .
$$

If $p_{g}(F) \geq 73$, then $d_{X} \leq 72\left(1+\frac{1}{73}\right) \frac{p_{g}(X)}{p_{g}(X)-3}<73$ if $p_{g}(X)>15987$, and the result follows in this case. Hence we can assume that $p_{g}(F) \leq 72$.

Let $r$ be the rank of the image of $H^{0}\left(f_{*} \omega_{X}\right) \otimes \mathcal{O}_{C} \rightarrow f_{*} \omega_{X}$. By [X, Proposition, p. 476], we have

$$
(g(C)-1)\left(p_{g}(F)-r\right) \leq r .
$$

If $r<p_{g}(F)$, by (3.3.2) we have $g(C) \leq r+1 \leq p_{g}(F) \leq 72$, which contradicts the assumption on $g(C)$ as in subsection 3.2. So we have $r=p_{g}(F)$. This implies that the restriction map $H^{0}\left(\omega_{X}\right) \rightarrow H^{0}\left(\omega_{F}\right)$ is surjective for the general fiber $F$ of $f$. So the restriction of $\phi_{X}$ to $F$ is just the canonical map $\phi_{F}$ of $F$.

Now let

be as in subsection 2.1. We have

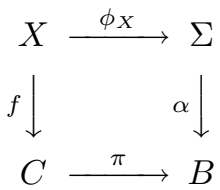

$$
d=\operatorname{deg} \phi_{F} \cdot \operatorname{deg} \pi .
$$

Note that

$$
\operatorname{deg} \phi_{F} \leq 9+\frac{27}{p_{g}(F)-2}
$$

by the the Miyaoka-Yau inequality.

Let $M\left(u, a, p_{g}(F)\right)$ be as in Proposition 2.8. We consider several cases according to $p_{g}(F)$.

If $3 \leq p_{g}(F) \leq 11$, by elementary calculations, we have

$$
M\left(2,\left[\frac{p_{g}(F)}{2}\right]+1, p_{g}(F)\right) \leq 297<g(C),
$$

where for rational number $x$, we define $[x]$ to be the largest integer $\leq x$. So the conditions of Proposition 2.8 are satisfied for $u=2, a=\left[\frac{p_{g}(F)}{2}\right]+1$ and $3 \leq p_{g}(F) \leq 11$. By Proposition 2.8 with $u=2$ and $a=\left[\frac{p_{g}(F)}{2}\right]+1$, we get

$$
\operatorname{deg} \pi \leq\left[\frac{p_{g}(F)}{2}\right]+1
$$

By (3.3.3), (3.3.4) and (3.3.5), we have $d \leq 72$.

Similarly, if $12 \leq p_{g}(F) \leq 23$ (resp. $24 \leq p_{g}(F) \leq 34$ ), by Proposition 2.8 with $u=6$ and $a=\left[\frac{p_{g}(F)}{6}\right]+4$ (resp. with $u=7$ and $a=\left[\frac{p_{g}(F)}{7}\right]+4$ ), we get $\operatorname{deg} \pi \leq\left[\frac{p_{g}(F)}{6}\right]+4$ (resp. $\operatorname{deg} \pi \leq\left[\frac{p_{g}(F)}{7}\right]+4$ ). Combining this with (3.3.3) and (3.3.4), we have $d \leq 72$.

Finally we assume that $35 \leq p_{g}(F) \leq 72$. In this case, we have

$$
M\left(20,36, p_{g}(F)\right) \leq M(20,36,72)=\frac{36 \cdot 1422}{35}<1463 .
$$


Since $g(C)=q(X) \geq 1463$ by the assumption (3.2), the conditions of Proposition 2.8 are satisfied for $u=20, a=36$ and $35 \leq p_{g}(F) \leq 72$. By Proposition 2.8 with $u=20$ and $a=36$, we get

$$
\operatorname{deg} \pi \leq 36
$$

By (3.3.1), we have $d_{X} \leq 72\left(1+\frac{1}{35}\right) \frac{p_{g}(X)}{p_{g}(X)-3}<75$, provided that $p_{g}(X)>\frac{2625}{11}$. So $d \leq 74$. We show that $d=74$ does not occur. Otherwise, by (3.3.3) and (3.3.4), we have either $\operatorname{deg} \phi_{F}=1$ and $\operatorname{deg} \pi=74 \operatorname{or} \operatorname{deg} \phi_{F}=2$ and $\operatorname{deg} \pi=37$; in both cases we get a contradiction by (3.3.6). Similarly, $d=73$ does not occur. This completes the proof of Theorem 1.1.

In the end of this section, we give some examples of 3 -folds with high canonical degree.

Example 3.4. Let $S$ be a smooth minimal surface with $\operatorname{deg} \phi_{S}=16$ (see [Pe, Example 5.8] for such surfaces), and let $C$ be a hyperelliptic curve of genus $g \geq 2$. Let $X=C \times S$. Then $X$ is a smooth minimal 3 -fold with $p_{g}(X)=3 g$ and $\operatorname{deg} \phi_{X}$ $=32$.

Remark 3.5. Let $S$ be a smooth minimal surface of general type. If the canonical map $\phi_{S}$ of $S$ is generically finite, then $\operatorname{deg} \phi_{S} \leq 36$, and the equality can only occur if $p_{g}(S)=3, q(S)=0, K_{S}^{2}=36$, and $\left|K_{S}\right|$ is base-point free (cf. (3.3.4)). A difficult problem is, does there exist a smooth surface with $p_{g}(S)=3, q(S)=0$, $K_{S}^{2}=36$, and $\left|K_{S}\right|$ being base-point free? An affirmative answer to this problem implies that there exist examples of 3 -folds of arbitrary large $p_{g}$ with deg $\phi_{X}=72$.

Example 3.6. Here are more examples of 3-folds of arbitrary large $p_{g}$ with $\operatorname{deg} \phi_{X}$ $=32$.

Let $n \geq 3$ be an integer. Let $C$ be a smooth curve of genus 3 , and $\eta \in P i c^{0} C$ a non-trivial 2-torsion point. Let $Y=C \times C \times \mathbb{P}^{1}$, and $p_{i}(i=1,2,3)$ the projections to its factors. Let $\delta=p_{1}^{*} \eta \otimes p_{2}^{*} \eta \otimes p_{3}^{*} \mathcal{O}_{\mathbb{P}^{1}}(n)$. Then the linear system $|2 \delta|=\left|p_{3}^{*} \mathcal{O}_{\mathbb{P}^{1}}(2 n)\right|$ is base-point free. Take $B \in|2 \delta|$ be a smooth divisor. Let $\pi: X \rightarrow Y$ be the morphism corresponding to the double cover data $(B, \delta)$. Then $X$ is 3 -fold with $p_{g}(X)=4(n-1)$ and $K_{X}^{3}=192(n-2)$. We have $\pi_{*} \omega_{X}=\omega_{Y} \otimes \delta \oplus \omega_{Y}$. Since $p_{g}(Y)=0, \phi_{X}=\phi_{\omega_{Y} \otimes \delta} \circ \pi$. Since $\omega_{Y} \otimes \delta=p_{1}^{*}\left(\omega_{C} \otimes \eta\right) \otimes p_{2}^{*}\left(\omega_{C} \otimes \eta\right) \otimes p_{3}^{*} \mathcal{O}_{\mathbb{P}^{1}}(n-2)$, we have that $\phi_{\omega_{Y} \otimes \delta}$ is the composition of the following morphisms:

$$
\begin{aligned}
& C \times C \times \mathbb{P}^{1} \stackrel{\phi_{\omega_{C} \otimes \eta} \times \mathrm{id}}{\longrightarrow} \mathbb{P}^{1} \times C \times \mathbb{P}^{1} \stackrel{\mathrm{id} \times \phi_{\omega_{C} \otimes \eta} \times \mathrm{id}}{\longrightarrow} \mathbb{P}^{1} \times \mathbb{P}^{1} \times \mathbb{P}^{1} \\
& \stackrel{\mathrm{id} \times \phi_{\mathcal{O}_{\mathbb{P}}(n-2)}}{\longrightarrow} \mathbb{P}^{1} \times \mathbb{P}^{1} \times \mathbb{P}^{1} \stackrel{\iota}{\longrightarrow} \mathbb{P}^{4 n-5},
\end{aligned}
$$

where $\iota$ is the Segre embedding. So $\operatorname{deg} \phi_{\omega_{Y} \otimes \delta}=16$ and hence $\operatorname{deg} \phi_{X}=32$.

Example 3.7 (3-folds of small $p_{g}$ with $\operatorname{deg} \phi_{X}=64$ ). Let $S$ be a numerical Campedelli surface (i.e., a smooth minimal projective surface of general type with $p_{g}=q=0$ and $K^{2}=2$ ) with $\left|2 K_{S}\right|$ being base-point free (see e.g., [Pe] for such surfaces), and let $C$ be a smooth curve of genus 3. Let $Y=C \times S$, and $p, q$ the projections to $C, S$, respectively. Let $\delta=p^{*} \eta \otimes q^{*} \omega_{S}$, where $\eta \in P i c^{0} C$ is a non-trivial 2-torsion point. Then the linear system $|2 \delta|=\left|q^{*} \omega_{S}^{\otimes 2}\right|$ is base-point free. Take $B \in|2 \delta|$ be a smooth divisor. Let $\pi: X \rightarrow Y$ be the morphism corresponding to the double cover data $(B, \delta)$. Then $X$ is 3-fold with $p_{g}(X)=6$ and $K_{X}^{3}=192$. We have that $\pi_{*} \omega_{X}=\omega_{Y} \otimes \delta \oplus \omega_{Y}$. Since $p_{g}(Y)=0, \phi_{X}=\phi_{\omega_{Y} \otimes \delta} \circ \pi$. 
Since $\omega_{Y} \otimes \delta=p^{*}\left(\omega_{C} \otimes \eta\right) \otimes q^{*} \omega_{S}^{\otimes 2}$, we have that $\phi_{\omega_{Y} \otimes \delta}$ is the composition of the following morphisms:

$$
C \times S \stackrel{\phi_{\omega_{C} \otimes \eta} \times \mathrm{id}}{\longrightarrow} \mathbb{P}^{1} \times S \stackrel{\mathrm{id} \times \phi_{\omega_{S}^{\otimes 2}}}{\longrightarrow} \mathbb{P}^{1} \times \mathbb{P}^{2} \stackrel{\iota}{\longrightarrow} \mathbb{P}^{5},
$$

where $\iota$ is the Segre embedding. So $\operatorname{deg} \phi_{\omega_{Y} \otimes \delta}=32$ and hence $\operatorname{deg} \phi_{X}=64$.

\section{ACKNOWLEDGMENTS}

The author is grateful to the referee for several helpful comments. This work has been supported by the NSFC (No. 10671003).

\section{REFERENCES}

Be. A. Beauville, L'application canonique pour les surfaces de type qénéral, Invent. Math. 55 (1979) 121-140 MR553705 (81m:14025)

Fu. T. Fujita, On Kaehler fibre spaces over curves, J. Math. Soc. Japan 30 (1978) 779-794. MR513085 (82h:32024)

Ha. C. Hacon, On the degree of the canonical maps of 3-folds, Proc. Japan Acad. 80 Ser. A (2004) 166-167 MR2099745 (2005f:14079)

M. Y. Miyaoka, The Chern classes and Kodaira dimension of a minimal variety, in Algebraic Geometry, Sendai, 1985, Adv. Stud. Pure Math. 10 North-Holland, Amsterdam, (1987) 449-476 MR946247 (89k:14022)

Mu-Sa. S. Mukai, F. Sakai, Maximal subbundles of vector bundles on a curve, Manuscripta Math. 52 (1985) 251-256 MR790801 (86k:14013)

P. U. Persson, Double coverings and surfaces of general type, in Algebraic geometry, LNM 687 (1978) 168-195 MR527234 (80h:14017)

Pe. C. Peters, On two types of surfaces of general type with vanishing geometric genus, Invent. Math. 32 (1976) 33-47 MR0409482 (53:13237)

X. G. Xiao, Algebraic surfaces with high canonical degree, Math. Ann. 274 (1986) 473-483 MR842626 (87g:14041)

Lmam, School of Mathematical Sciences, Peking University, Beijing 100871, PeoPLE'S REPUBLIC OF ChinA

E-mail address: jxcai@math.pku.edu.cn 